\title{
Algebraic fields and the dynamical approach to physical geometry
}

\author{
Tushar Menon* \\ Balliol College, University of Oxford. \\ University of Illinois at Chicago.
}

\section{Contents}

1 Introduction $\quad 1$

2 The dynamical approach $\quad 3$

3 Algebraic fields $\quad 4$

4 Norton's criticism $\quad 5$

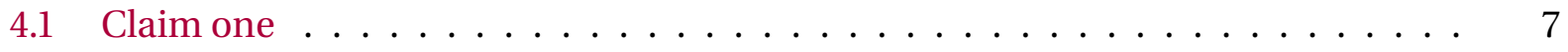

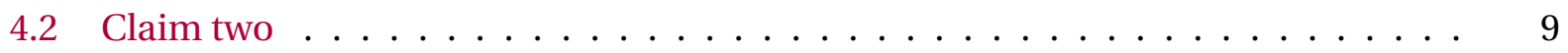

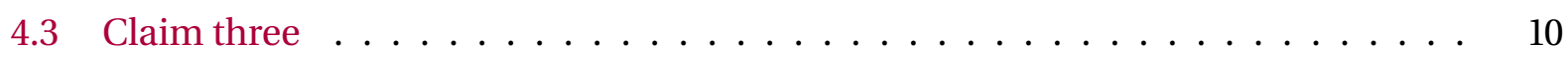

5 Conclusion 11

\section{Introduction}

The first decade of the twenty-first century brought with it a series of publications by Harvey Brown and Oliver Pooley [2, 3, 4] in which they bestowed upon special relativity (SR) a novel position regarding its ontology. Distinct from the substantivalism-relationalism debate, whose subject matter concerned the fundamentality of spacetime points, the ensuing dynamical-geometrical debate was over the ontological status of the metric field. According to Brown and Pooley's papers, the metric tensor of SR plays nothing more than a codificatory role in the dynamics. Additionally, it is itself a 'glorious non-entity'.

Their dynamical approach to physical theories zeroes-in on what had been, in their view, an unexplained correlation between facts about geometry and facts about the behaviour of dynamical

*email: tushar.menon@balliol.ox.ac.uk 
fields (such as those constitutive of rods and clocks). The position asserts, among other things, that facts about physical geometry are grounded in, or explained by, facts about dynamical fields, not the other way round. The converse position-that geometry is explanatory of matter field behaviour-is ubiquitous, and is the orthodox position on physical geometry and spacetime structure. One of the most persistent critics of the dynamical view, John Norton [10], is taken to have articulated a robust objection. His claim is that the proponent of the dynamical approach, the so-called constructivist, to use Norton's neologism, is illicitly committed to spatiotemporal presumptions in 'constructing' spacetime from facts about dynamical symmetries.

The image of construction brings with it an image of building blocks. The standard way of representing (at least some of) the building blocks of a theory is in terms of kinematical structure. Broadly speaking, this is the structure that needs to be presupposed in order to build models of physical goings-on. In spacetime theories, this structure is represented using kinematically possible models (KPMs). KPMs of relativistic theories are tuples of the form $\left\langle M, g_{a b}, \phi^{i}\right\rangle$, where $M$ is a smooth manifold, $g_{a b}$ is a Lorentzian metric tensor and $\phi^{i}$ is a placeholder for matter fields. A KPM of SR, then, is one in which $g_{a b}=\eta_{a b}$, the Minkowski metric.

Models of this kind implicitly (and illicitly) have an extra bit of structure-a link between the manifold and the fields. Specifically, for any point in $M$, distinct fields, $\phi_{1}, \ldots, \phi_{n}$, can be 'evaluated' at that point, and their values can then be taken, as e.g. Field does [5], to represent properties of the same spacetime point. Call a KPM in which this link does exist a KPM of the first kind. Constructing KPMs in this way is so intuitive that it is easy to lose sight of the fact that nothing in the mathematical structure of the model makes the link necessary; elements of the set $M$ do not necessarily have primitive identity. To be sure, I make no commitment to whether or not it is mathematically necessary that the points of a set have some primitive distinguishing feature. All I claim is that one must be aware of whether or not such an assumption is being made at the mathematical level, and if so, what its justifications might be. In what follows, we will be able to interpret Norton's physical requirement justifying his commitment to the primitive identity of mathematical points in the manifolds in his models; in \$3, I demonstrate how the notion of a point, even mathematically, can be non-fundamental, and therefore devoid of primitive identity.

When we express fields as 'functions of spacetime', in the form ' $\phi(x)$ ', they are composite functions acting on a set of points, $M$, of the form $\phi \circ x: M \rightarrow \mathbb{V}$, where $\mathbb{V}$ is some space in which the fields take their values. There is a notational ambiguity here. It is sometimes the case that ' $x$ ' is used to represent the coordinate function assigned to a point in $M$, its value, and also the preimage of a point in $\mathbb{R}^{n}$ under that function. In this paper, I use ' $x$ ' to represent the function, and $p$ to represent the preimage of a point in $\mathbb{R}^{4}$ under $x$.

Given the arbitrariness of the coordinate function, there is no a priori need for the coordinatisations of two separate composite functions to coincide. In other words, there is no a priori reason to require that the $x$ in $\phi(x)$ is the same as the $x$ in $\psi(x)$-indeed, our use of the same variable to denote both is indicative of a failure to allow for this mismatch. Call models in which the preimages of $x$ in $\phi(x)$ and $\psi(x)$ can represent different points, KPMs of the second kind. As 
we will see in $\$ 4$, Norton’s criticism is based on assuming the KPMs at play can only be of the first kind. The central aim of this paper is to demonstrate that KPMs of the second kind can be constructed for SR. And insofar as this is the first approach to spacetime theories that allows for such a construction, I contend that the dynamical approach, pace Norton, is a genuinely novel approach to spacetime.

\section{The dynamical approach}

The dynamical approach to SR takes seriously the fact that measuring devices like rods and clocks are themselves entities whose constituent matter fields are governed by the same dynamical laws as the systems they measure. Consequently, any explanation of their behaviour must make reference to facts about the dynamical equations that govern them. Falling back on a geometrical explanation of the form 'rods and clocks contract because they are embedded in Minkowski spacetime' is, in the eyes of the proponent of the dynamical approach, unsatisfactory, unless the geometry itself is taken to be a convenient shorthand for the symmetry structure of the dynamical field equations.

A specially relativistic field theory is standardly presented in terms of dynamically allowed maps from a manifold into some mathematical space. The metric structure on the Minkowski manifold encodes certain facts about dynamical symmetries-these are transformations on the fields that leave the form of the dynamical equations unchanged. According to the dynamical approach, the metric structure attributed to this manifold is nothing more than a reflection of this dynamical structure of matter fields. In particular, there is no sense to be made of the claim that the Minkwoski manifold constrains or explains the symmetry structure of the dynamical laws. Further, the position engenders an ontological claim-the Minkowski metric is thought of as a 'glorious non-entity' [4], in the sense that it is not ontologically independent of matter fields (this is not to say that proponents of the dynamical approach are anti-realists about it; just anti-fundamentalist).

This raises an immediate question: even if one accepts that the metric is a non-fundamental entity, what is the status of the smooth manifold? After all, the smooth manifold is the space of independent variables in terms of which the field theory is expressed. If that too is a non-entity, then how can one speak coherently of a field theory? How can one even define a field? Pooley's response [11, \$6.3.2] is that theirs was never intended to be a reductive story about all putatively 'spatiotemporal' structure. They still presuppose the existence of a smooth manifold, with its attendant topological properties. The dynamical symmetries of the fields then ground the further metric structure of the manifold. Some readers might find themselves unmoved by this response. My response in this paper, therefore, is to extend the dynamical approach to account for the existence of topological and smooth structure on the manifold. 


\section{Algebraic fields}

If we think of the ontology of a generic field theory as being represented by mathematical objects, then we have a nontrivial task in accounting for what the relationship between mathematical objects and the putative physical objects they represent actually is. The task is nontrivial because of certain artefacts of the language which we use to formulate our theories, the symmetry transformations, which disrupt a straightforwardly bijective mapping between mathematical objects and worldly entities. Consider a collection of models of the form $\left\langle M, \eta_{a b}, \phi^{i}\right\rangle$, where $M$ is a smooth, compact, Hausdorff manifold, $\eta_{a b}$ is the Minkowski metric tensor and $\phi^{i}$ is a placeholder for all the fields in the theory, $\phi(x), \psi(x), \chi(x) \ldots$, etc., which satisfy a prescribed set of Lorentz-invariant dynamical equations. ${ }^{1}$ On this setup, the matter fields are represented as functions, $\phi(x)$, from the manifold, $M$ (recall that this is shorthand for $\phi \circ x$ ), to some mathematical space. Following Huggett, [8], call this sort of representation of matter a material field.

There is a different, but entirely equivalent way of setting up a Klein-Gordon theory. Consider the set of all smooth real-valued functions defined on some compact, Hausdorff manifold $M$. This set is usually denoted as $C^{\infty}(M)$. Being real-valued, these functions assign, to each point, some real number. As a result, there is a natural way to talk about the addition of two functions, $\phi(x)$ and $\psi(x)$ - simply add their values at every point. The notation ' $\psi(x)$ ' is, unfortunately, ambiguous between the value assigned to some point under the mapping, $\psi$, and the abstract object that defines the mapping rule. I will use $\psi(x)$ to represent the function as a material field, and $\psi$ to represent the abstract function.

It can easily be shown that the set of smooth functions, $C^{\infty}(M)$, under the operations of addition and multiplication so defined, is an algebra over the field of real numbers. This can be given a wholly algebraic characterisation as an automorphism on this algebra (which satisfies the Leibniz rule). Of course none of this is intended to argue for a shift in the metholodogy associated with finding these derivative operators. All that changes is our understanding of what the sort of mathematical object our experimental data gives us reason to believe in. Whereas earlier we might have thought of the Klein-Gordon operator as a differential operator on a manifold and stopped there, now it should be thought of as representing some preferred set of automorphisms on $C^{\infty}(M)$.

The abstract algebra of smooth functions, $C^{\infty}(M)$, together with this derivative operator contains enough structure to allow us to completely characterise the dynamical scalar field system. In particular, the fields $\psi$ and $\phi$ no longer need to be defined as functions on a manifold-they

\footnotetext{
${ }^{1}$ I should, at the outset, highlight a theoretical (though not practical) limitation of the analysis provided in this chapter-attention here is restricted to compact manifolds. The compactness condition is non-standard; indeed, Minkowski spacetime is non-compact. The algebraic analysis of spacetime points presented in this and the following section, depends on the validity of the Gel'fand-Naimark theorem, which applies only to continuous functions on a compact, Hausdorff manifold. The construction, therefore, reproduces a proper subset of SR models. This is a feature, not a bug, of this account. Assumptions about the asymptotic behaviour of fields are never justified within the theory itself. Thus, for any particular collection of fields of interest, we can consider the associated manifold to be a compact submanifold of $M$, and then run the argument provided in this paper unproblematically.
} 
can be thought of merely as elements of an abstract algebra which may, but need not, be realised as smooth functions on a manifold. Call this non-manifold-based representation of matter an algebraic field, and the set of algebraic fields picked out by the derivative operator the space of dynamically possible fields. It is important to point out that there is no sense to be made of the 'value of the field at a point' on the algebraic field approach. Here, the primary object is the entire field, conceived of as a single element in an algebra.

Let us pause here to ensure that we haven't lost sight of the physical facts that we are trying to capture. The material field picture of the world is relatively close to the manifest image, at least on our simple scalar field theory (imagine that $\phi$ is a temperature field). It captures our intuitions about a pre-existing set of spacetime points which 'contain' the scalar field. I contend that this intuition is misleading - nothing in our physics warrants taking $M$ rather than $C^{\infty}(M)$, or structure defined in terms of it, as primitive. On the algebraic field view, all we are doing is taking the field to be primitive.

If the abstract algebra out of which the dynamically possible algebraic fields are constructed admits a realisation as a set of material scalar fields, then all of the information about the underlying manifold - its topological and smooth structure-is already contained in the abstract algebra. This fact is important-if we merely consider the vector space, $C_{V}^{\infty}(M)$ that consists of the smooth functions from a manifold, then there is not enough information there to uniquely determine the topology of the base manifold. This remarkable result, establishing the complete equivalence of Hausdorff topological spaces and commutative $C^{*}$ algebras, was demonstrated by Gel'fand and Naimark [6], and is referred to as the Gel'fand-Naimark theorem.

This characterisation of the theory is no more abstract than the model-theoretic characterisation. We therefore see that the manifold is no more required to be part of that description, for the algebraic formalism to make sense, than four concrete objects are required in order to make sense of $2+2=4$. In other words, the abstract algebraic picture should not be thought of as less able to capture the important structural dynamical features of a theory like SR. It is merely the case that it does not lend itself to a (relatively) simple visualisation of the ontology in the way that the manifold picture does. Our physical theories, however, consist of much more structured mathematical objects than just scalars - they include vectors, tensors, connections, and so on. Geroch's classic paper on Einstein algebras [7] demonstrates that all of these objects can be characterised algebraically as well.

\section{Norton's criticism}

Norton claims that the dynamical approach to relativity (or, to switch to his term, constructive relativity) fails. He advances the following criticism of the constructive relativist project: that it 'only succeeds if constructivists antecedently presume the essential components of a realist conception of spacetime' [10, p. 821]. Norton is explicit about what, for him, constitutes a realist conception of Minkowski spacetime: 
(1) There exists a four-dimensional spacetime that can be coordinatized by a set of standard coordinates $(x, y, z, t)$ related by the Lorentz transformation.

(2) The spatiotemporal interval $s$ between events $(x, y, z, t)$ and $(X, Y, Z, T)$ along a straight [footnote suppressed] line connecting them is a property of the spacetime, independent of the matter it contains, and is given by

$$
s^{2}=(t-T)^{2}-(x-X)^{2}-(y-Y)^{2}-(z-Z)^{2}
$$

(3) Material clocks and rods measure these times and distances because the laws of the matter theories that govern them are adapted to the independent geometry of this spacetime. [10, p. 823]

Before dealing with the specific challenges Norton levels against the constructive project, it is worth addressing two ways in which this conception of spacetime realism misrepresents the constructive project's aims (insofar as it asserts that the constructive project must deny them). First, that the constructivist must deny the existence of a 'four-dimensional spacetime that can be coordinatized by a set of standard coordinates.' Nothing in the setup of the dynamical approach commits its adherents to the denial of the existence of spacetime-all that is required is that spacetime structure (to the extent that it can be identified) be reducible to facts about the dynamics of fields. In other words, the constructivist need only be committed to the non-fundamentality of spacetime, in the sense that it is ontologically dependent on matter fields.

Second, that the spatiotemporal interval is a 'property of the spacetime independent of the matter it contains.' This notion of independence is ambiguous. It could either mean (i) that the interval is a property of spacetime that does not require matter fields in order to be intelligible (call this the ontological reading) or (ii) that the interval still requires matter fields in order to be intelligible, but is neutral with respect to idiosyncratic details of which of several distinct matter fields is being considered (call this the epistemological reading). The constructivist denies the ontological reading, but accepts the epistemological reading. Thus the spacetime realist position that the constructive denies is more accurately characterised as follows:

(1) There exists a fundamental four-dimensional spacetime that can be coordinatized by a set of standard coordinates $(x, y, z, t)$ related by the Lorentz transformation.

(2) The spatiotemporal interval $s$ between events $(x, y, z, t)$ and $(X, Y, Z, T)$ along a straight [footnote suppressed] line connecting them is a property of the spacetime, ontologically independent of the matter it contains, and is given by

$$
s^{2}=(t-T)^{2}-(x-X)^{2}-(y-Y)^{2}-(z-Z)^{2}
$$

(3) Material clocks and rods measure these times and distances because the laws of the matter theories that govern them are adapted to the ontologically independent geometry of this spacetime. 


\subsection{Claim one}

The construction project must tacitly assume an already existing spacetime endowed with topological properties, so that it can introduce spatiotemporal coincidences, and a unique set of standard coordinates $(x, y, z, t)$. [10, p. 824]

Norton, here, assumes that no sense can be made, in purely constructive terms, of a spatiotemporal coincidence, as a result of which, it needs to be presupposed. This is not true. As we will see in what follows, Norton conflates a difficulty in having epistemic access to spacetime points with an inability to define them; the constructivist can perfectly easily make sense of an appropriate definition of a spacetime point, regardless of whether its interpretation as a point of coincident field values is dynamically accessible.

On the algebraic view, the notion of a spacetime point is derivative-it is specified by some linear functional, say $X$ on $C^{\infty}(M)$, which maps $\phi$ to an element in the space of maximal ideals of $C^{\infty}(M) .{ }^{2}$ Let $\mathfrak{M}$ represent the space of maximal ideals-for a compact manifold, each element is the set of functions which vanish at $p$. We thus see that each spacetime point is uniquely associated with an element of $\mathfrak{M}$. More generally, any closed subset of the manifold is associated with a closed ideal.

If we had a more complicated theory, say, some interacting theory of a vector field and a connection (like electromagnetism in the vector potential formalism), the equivalent of spacetime points can still be built out of the algebra $C^{\infty}(M)$-this always remains a constituent of the KPMs. Then separately, using the algebra $C^{\infty}(M)$ one can construct derivatives and tensors in the standard way-these are then represented as elements $\phi$ of some solution space.

Thus, $X(\phi)$ just gives us the quadruple $\left(x^{\mu}\right)$, the image of the function $x$, that we would have expressed $\phi$ in terms of, had we chosen to realise it as a scalar field on a manifold. In order to see if it coincides with, say, $X(\psi)$, where $\psi \in \mathfrak{B}$ is a different dynamical field coupled to $\phi$, it suffices to check the space of dynamically possible models of the interacting theory. If $X(\phi)=X(\psi)$, then we have defined the spatiotemporal point of coincidence of the two fields. Whether this definition is useful, of course, depends on details of the dynamics.

Consider, now, a coordinate system on which we have good reason to believe that, say, a Gaussian wave packet of the $\phi(x)$ field bounced off a Gaussian wave packet of the $\psi(x)$ field in the neighbourhood of some point. There will be a class of coordinatisations which assign field values in such a way that the dynamics of each field determines that a collision took place in the vicinity of some point, and another class of coordinatisations on which the kinks in the trajectories (or, more generally, some fact about the dynamical interaction) of each particle do not take place at the same coordinate value. On pragmatic grounds, the former class of coordinate systems will be preferable. For free fields, there simply is no operational sense of spatiotemporal point coincidence, although such points can still be defined. In such a case, though, these would amount to arbitrary stipulations.

\footnotetext{
${ }^{2}$ Recall that, for simplicity, we are dealing with linear dynamics of scalar fields, so the space of DPMs has the structure of a vector space. $X$ is only a linear functional in cases like this. More generally, $X$ is just a smooth mapping.
} 
This observation points to an arbitrariness in our definitions of 'free' and 'interacting' particles. The trajectory of a particle of the $\phi$ field might be such that it contains a change in direction that, on an interacting field picture, we attribute to it having collided with another field, $\psi$ at some point. That is to say, there is a choice of functional $X$ such that $X(\phi)=X(\psi)$ at the appropriate point (actually infinitely many). Of course, there are also infinitely many choices of $X$ on which $\phi(x)$ suddenly has a kink in its trajectory at some point, and $\psi(x)$ does too, at a different point. We can always absorb these kinks into a complicated dynamical picture of free fields, and we lose no descriptive power. But on this picture, each field, in a sense 'inhabits its own spacetime'.

When thinking about field theories in this algebraic sense, several standardly intuitive concepts, such as energy or time evolution, are no longer necessary or even applicable. Let the space of dynamically possible fields be represented by $\mathfrak{A}$. Each element, $\phi \in \mathfrak{A}$, is the equivalent of a complete history of a material field, and the notion of, for example, a Hamiltonian generating time evolution of data only becomes meaningful in the context of a specific solution in a specific realisation-a Cauchy problem on the material field realisation. If we consider elements of the algebra to be the fundamental entities, then spacetime points become derivative, independently of the form that the dynamics might take.

In such a theory, the means of having epistemic access to a particular point (or arbitrarily small region of spacetime) is through the interaction dynamics-but this is not how we need to define a point. And Norton's criticism of the constructivist is based on their purported inability to define a point derivatively.

Of course, this is only part of the story for the constructivist-in order to recover the fact that spatiotemporal coincidences are preserved under homeomorphisms, it must be the case that the dynamical symmetries, encoded in the relevant derivative operator, preserve spatiotemporal coincidences. In other words, it must be the case that the dynamically possible models are such that under the automorphisms, $S: \mathfrak{A} \rightarrow \mathfrak{A}, S: \mathfrak{B} \rightarrow \mathfrak{B}$, it is the case that if $X(\phi)=X(\psi)$, then $X S(\phi)=X S(\psi)$.

The fact that the dynamical symmetry group of SR, the Poincaré group, is a Lie group (which acts freely and transitively) ensures that this is the case. The dynamical symmetry group acts on states-elements of $\mathfrak{A}$ which, to the extent that they can represent approximately particle-like behaviour, are trajectories in $M$. All Lie groups are smooth manifolds-which means that their topology is the standard topology. This is the topology that the space of solutions inherits.

According to the Gel'fand-Naimark theorem, the space, $\mathfrak{M}$, of closed maximal ideals of the algebra $C^{\infty}(M)$ is homeomorphic to $M$. The set $\mathfrak{X}$, of linear functionals $X$, determines $\mathfrak{M}$, when its elements act on any element of $C^{\infty}(M)$. Thus the set $\mathfrak{X}$ of linear functionals determines the manifold of 'spacetime' points. Any automorphism on $\mathfrak{A}$ and $\mathfrak{B}\left(\subset C^{\infty}(M)\right)$ that is itself an element of a Lie group will preserve the coincidence $X(\phi)=X(\psi)$-this is just another way of saying a Lie group element generates a diffeomorphism on the underlying manifold of the space on which it acts. Note that this does not require that $\mathfrak{A}$ be a subalgebra of $C^{\infty}(M)$, or even a vector space (although, for dialectical simplicity, I have taken this to be the case in this example). The algebraic 
properties of $\mathfrak{A}$ are determined by the form of the derivative operator, $D$, while the (independent) topological and differential properties of $\mathfrak{A}$ are determined by its dynamical symmetry group.

This is not the same as using the energy of interactions from, say, the spin-coupling term of the Hamiltonian to designate the same spacetime event, a point Norton himself raises. There is no reference to energy in the proposal here. Rather, what is accounting for the coincidence of $X(\phi)$ and $X(\psi)$ is that the values of these functionals are preserved by a certain class of automorphisms on $\mathfrak{A}$ and $\mathfrak{B}$.

In short, the kinematical structure is provided by the algebra $C^{\infty}(M)$, and mathematical objects defined therefrom. The kinematical structure can be used to define a set of linear functionals (on $C^{\infty}(M)$ ) that maps any element to a set, $\mathfrak{M}$, of maximal ideals. Because the topological and differential structure of $M$ is encoded in $C^{\infty}(M)$-call this the kinematical smooth structure - this is equivalent to choosing a smooth manifold, $M$. The tensorial objects constructed out of $C^{\infty}(M)$ will always form a vector space, but the subset of 'dynamically allowed' objects, picked out by some derivative operator need not. At the level of kinematics, this suffices to define KPMs of the second kind.

The constructivist can, thus, talk about non-fundamental spacetime point coincidences (whose utility is determined by dynamical considerations) that are preserved under homeomorphisms. All of this can be done without having to presuppose fundamental, ontologically independent spatiotemporal facts. Norton assumes that there is no way of writing down a geometrical theory without points. If this had been true, then his challenge to the constructivist would have been devastating. But using Gel'fand and Naimark's result to refine our notion of kinematical structure, Norton's first claim, that 'attempts to extend [...] constructivism to spacetime points will fail' $[10$, p. 829] can be easily shown to be false.

\subsection{Claim two}

The Lorentz covariance of all matter theories asserts an adaptation between matter and this spacetime akin to the realist's [(3)], although without [(3)]'s presumption of the direction of the adaptation of matter to spacetime. [10, p. 824]

This second criticism loses all of its bite once it has been shown that spacetime points do not have to be presupposed by the constructivist. Once one has constructed a smooth manifold in the manner described in \$3, one can then use Brown and Pooley’s truncated Lorentzian pedagogy, realised either in Humean terms, as Pooley [11] and Stevens [12] do, or in Kleinian geometric terms as Wallace [13] does, in order to account for the appearance of Minkowski metric structure. This structure is then merely a reflection of the symmetry properties of dynamical fields. In that sense, the (metric) structure of spacetime is, indeed, adapted to the matter fields in virtue of having been derived from them, and it could not have been any other way. There is no 'direction of adaptation' between spacetime and matter any more than there is a direction of adaptation between 'bachelor' and 'unmarried man'. 


\subsection{Claim three}

Constructivists must accept that spatial distances and times elapsed are properties of spacetime as asserted in [(2)], on pain of failing to reconstruct traditional spacetime geometry and also having to accept an extreme form of operationalism in which quantities have values only if they are actually measured. [10, p. 824]

To bolster his claim that the constructivist is committed to 'an extreme form of operationalism', Norton presents an example of a region of spacetime that is either (A) devoid of matter or (B) hosts a static matter distribution. On this, Norton offers the following:

In this part of spacetime, we can select two noncoincident timelike-separated events A and B such that nothing changes as we pass along the straight segment of spacetime connecting them. In the ordinary realist's conception, we would say that some time elapses between them. What can a constructivist say? There are no material clocks actually present measuring the time elapsed, for there is either no matter present or no change in the matter present as we pass from A to B. So the constructivist has no material basis for the recovery of a time change. If times elapsed are to supervene on matter, or more vaguely to be a result of the properties of matter, then the absence of any change in the matter entails that there is no change in times elapsed. [10, pp. 831832]

There is a subtle but important difference between the case of 'no matter' and 'static matter'. In the former case, as Pooley points out, 'for the constructivist there is literally nothing in an empty region and so nothing whose geometrical properties might be indeterminate. The constructivist does not believe in the existence of an independently existing spacetime!' [11, §6]. There are no matter fields, a fortiori no dynamics, a fortiori no structure to preserve in the algebra $C^{\infty}(M)$. Further, there is no reason to believe that the space of KPMs should refer to $C^{\infty}(M)$ rather than, say, $C^{\infty}\left(M^{\prime}\right)$, where $M ¥ M^{\prime}$. So even the point-structure to be specified is totally arbitrary-every choice of kinematical structure is as good as every other.

In the latter case, we find ourselves in a position alluded to at the end of $\$ 4.1$. On the algebraic picture, the fundamental object is the entire field - to the extent that we are interested in 'regions', we must talk about closed ideals of the algebra $C^{\infty}(M)$. These ideals will still specify topological information about 'regions', but will be silent on metric structure-the Gel'fand-Naimark theorem only recovers topological and smooth-structure. Since there is no interacting field, there is no choice of linear functionals that is dynamically privileged, so all sets of such functionals are equally useful in realising the algebraic field as a material field. On the material field realisation, therefore, in static regions, there will simply be no fact about how much proper time has elapsed between any two points. Indeed, this functions as reductio against Norton's assumption that there is a determinate fact about the (proper) time that has elapsed between A and B. 


\section{Conclusion}

In this paper, I presented an extended version of the dynamical approach, based on the observation that the kinematically possible models can be characterised without requiring primitive spacetime point-coincidence of fields. Once we are aware of the implicit link in our KPMs between spacetime points and 'points of independent variable coincidence of field values', it is important to determine whether or not this structure is epistemically warranted and dynamically necessary. I argued that it is neither.

Using an algebraic reformulation of classical field theories, which did away with the kinematical requirement that fields be defined as maps from manifolds, I demonstrated how 'KPMs of the second kind' could be constructued. These KPMs (and their associated Poincaré-invariant DPMs) were then be used to counter Norton's criticism of the dynamical approach.

\section{References}

[1] Pablo Acuña. Minkowski spacetime and lorentz invariance: The cart and the horse or two sides of a single coin? Studies in History and Philosophy of Science Part B: Studies in History and Philosophy of Modern Physics, 55:1-12, 2016.

[2] Harvey R Brown. Physical Relativity. Oxford University Press, Oxford, 2005.

[3] Harvey R Brown and Oliver Pooley. The origin of the space-time metric: Bell's 'lorentzian pedagogy'and its significance in general relativity. In Craig Callender and Nick Huggett, editors, Philosophy Meets Physics at the Planck Scale. Cambridge University Press, Cambridge, 2001.

[4] Harvey R Brown and Oliver Pooley. Minkowski space-time: a glorious non-entity. Philosophy and Foundations of Physics, 1:67-89, 2006.

[5] Hartry Field. Can we dispense with space-time? In PSA: Proceedings of the Biennial Meeting of the Philosophy of Science Association, volume 1984, pages 33-90. Philosophy of Science Association, 1984.

[6] I.M Gelfand and M.A Naimark. On the embedding of normed rings into the ring of operators in hilbert space. Mat. Sbornik, 12:197-213, 1943.

[7] Robert Geroch. Einstein algebras. Communications in Mathematical Physics, 26(4):271-275, 1972.

[8] Nick Huggett. Non-commutative spacetime. Unpublished Manuscript, 2016.

[9] Wayne C Myrvold. How could relativity be anything other than physical? Studies in History and Philosophy of Science Part B: Studies in History and Philosophy of Modern Physics, 2017.

[10] John D Norton. Why constructive relativity fails. The British journal for the philosophy of science, 59(4):821-834, 2008.

[11] Oliver Pooley. Substantivalist and relationalist approaches to spacetime. In Robert W Batterman, editor, The Oxford Handbook of Philosophy of Physics. Oxford University Press, 2013. 
[12] Syman Stevens. The dynamical approach as practical geometry. Philosophy of Science, 82(5):1152-1162, 2015.

[13] David Wallace. Who's afraid of coordinate systems? an essay on the representation of spacetime structure. Studies In History and Philosophy of Science Part B: Studies In History and Philosophy of Modern Physics, 2017. 